\title{
Robust Iterative Learning Control for a Class of Linear Systems with Data Dropouts
}

\author{
Wang Rui, Ma Xinghe, and Bu Xuhui \\ School of Electrical Engineering \& Automation, Henan Polytechnic University, Jiaozuo 454000, China \\ Correspondence should be addressed to Wang Rui; wangrui_hpu@126.com
}

Received 26 March 2013; Accepted 10 July 2013

Academic Editor: Yingwei Zhang

Copyright (C) 2013 Wang Rui et al. This is an open access article distributed under the Creative Commons Attribution License, which permits unrestricted use, distribution, and reproduction in any medium, provided the original work is properly cited.

\begin{abstract}
A robust iterative learning control algorithm is proposed for a class of intermittent systems with disturbances and uncertain initial conditions. Based on the contraction mapping approach, the convergence condition for the proposed algorithm is first given, and then the bounds on control input and output trajectories can be obtained. It is shown that these bounds depend on bounds on the initial condition errors and disturbances, and the bounds are zero in the absence of these disturbances. A numerical example is also given to verify the theoretical result.
\end{abstract}

\section{Introduction}

Iterative learning control (ILC) is an attractive technique when it comes to dealing with systems that execute the same task repeatedly over a finite time interval. The key feature of this technique is to use information from the previous (and/or current) operation (or iteration) in order to enable the controlled system to perform progressively better from operation to operation. This technique has been the center of interest of many researchers over the last two decades [1-6]. Robustness is an important issue for control approaches [79], especially in practical systems. Hence, the robustness for ILC has been discussed in several aspects $[3,4,6]$.

On the other hand, networked control systems (NCSs) are also the focus of many research studies over the last few decades [10-13]. Compared to the conventional pointto-point system connection, NCSs have the advantages of easy installation and reduced setup, wiring, and maintenance costs. In NCSs, data can travel through the communication channels from the sensors to the controller and from the controller to the actuators. Data packet dropout (a kind of uncertainty) is a common problem in networked control systems and could happen due to node failures or network congestion. Because of random dropout, conventional methods for estimation and control cannot be used directly.

The data dropout problem in the context of ILC has been studied in [14-19], and such an ILC system is a call intermittent ILC system. In [14, 15], an optimal learning gain matrix is given for intermittent ILC systems. They considered the problem where each component in the multivariable output vector of the plant is subject to a dependent or independent dropout, respectively. In [16], an averaging ILC algorithm is proposed to overcome the random data dropout, and it is shown that such an ILC algorithm can perform well and achieve asymptotic convergence in ensemble average along the iteration axis. In [17], the convergence of first-order ILC and high-order ILC for linear intermittent ILC systems is considered. Using the super-vector technique, such an ILC system can be modeled as an asynchronous dynamical system with rate constraints on events in the iteration domain, and then the convergence condition can be provided by solving a binary linear matrix inequality. It is worth noting that the binary linear matrix inequality is difficult to be solved, especially for the high order ILC scheme. To avoid the problem, another convergence condition in the expectation sense is given in [18]. In [19], the issue of intermittent ILC is considered for a class of nonlinear systems. Key conclusions of these works are that the ILC systems can still guarantee convergence in the face of data dropout as long as there is not a $100 \%$ dropout.

All the aforementioned papers are considered intermittent ILC systems under the two common assumptions, identical initial condition and iteration-independent disturbances. However, in the practical implementation, the initial 
condition is often uncertain, and the disturbance is also stochastic, nonrepeating disturbances, such as measurement disturbance (or measurement noise). Hence, it is necessary to understand exactly how disturbances and uncertain initial conditions affect the intermittent ILC process and to develop ILC algorithms that are robust to their presence. This paper proposes a robust ILC design approach for intermittent ILC systems in the presence of both uncertain initial conditions and nonrepeating disturbances. After introducing a forgetting factor, the improved ILC algorithm can obtain a better performance in handling initial shifts and disturbances, while the P type ILC obtains the large tracking errors.

The remainder of this paper is organized as follows. In Section 2, the problem formulation is first described, and then a robust ILC law is proposed. In Section 3, a sufficient condition which guarantees the stability of the intermittent ILC system is given. In Section 4, an example is presented to validate the theoretical result. Finally, some conclusions are given in Section 5.

\section{Problem Formulation}

Consider the following linear discrete-time system:

$$
\begin{gathered}
x_{k}(t+1)=A x_{k}(t)+B u_{k}(t)+w_{k}(t), \\
y_{k}(t)=C x_{k}(t)+v_{k}(t)
\end{gathered}
$$

where $k$ denotes the iteration number and $t \in[0, N]$ is a finite time interval. $x(t) \in R^{n}$ is the state vector, $u(t) \in R^{p}$ is the input vector, and $y(t) \in R^{m}$ is the output vector, $A \in$ $R^{n \times n}, B \in R^{n \times p}, C \in R^{m \times n} \cdot w_{k}(t), v_{k}(t)$ are bounded state and output disturbance.

The following assumptions for the linear system (1) are given.

Assumption 1. For a given desired trajectory $y_{d}(t)$, there exists a desired control input $u_{d}(t)$ such that

$$
\begin{gathered}
x_{d}(t+1)=A x_{d}(t)+B u_{d}(t), \\
y_{d}(t)=C x_{d}(t),
\end{gathered}
$$

where $x_{d}(t)$ is the desired state and $u_{d}(t)$ is the desired input.

Assumption 2. The disturbances are bounded in sense of $\left\|w_{k}(t)\right\|<b_{w},\left\|v_{k}(t)\right\|<b_{v}$, where $b_{w}, b_{v}$ are positive constants.

Assumption 3. All operations start within a neighborhood of $x_{d}(0)$ in the sense of $\left\|x_{k}(0)-x_{d}(0)\right\|<b_{x_{0}}$, where $b_{x_{0}}$ is positive constant. This assumption is natural in many repetitive dynamic systems such as the repeatability in robot specifications.

The control target is to find a control input sequence $u_{k}(t)=u_{d}(t)$, such that $y_{k}(t)$ converges to $y_{d}(t)$ as $k \rightarrow$ $\infty$; that is, as the learning iteration repeats, the system output converges to the desired trajectory. It is assumed that the ILC algorithm is implemented via a networked control system. That is, the sensor, actuator, and the linear system are remotely controlled by an iterative learning controller that interchanges output measurement and control signals through a communication network. In the sensor and controller sides, they are time driven, and in the actuator side it is event driven. The network can be modeled as a switch that opens and closes in a random manner. When a switch is open, its output is held at the previous value, and the data packet is lost. As mentioned in [10-13], there are two different types of data dropouts in networked control systems. This paper only considers the output measurement dropout. The system output $y_{k}(t)$ is passed through the network, and there may be random dropouts. Thus, the current observation $\tilde{y}_{k}(t)$ is the controller received output, which is the system output $y_{k}(t)$ with the probability of $\bar{\alpha}$. In the case of no new data, previous data will be used, so the previous data, $y_{k-1}(t)$, will be used with the probability of $1-\bar{\alpha}$. These can be represented by the following relations:

$$
\tilde{y}_{k}(t)=\alpha y_{k}(t)+(1-\alpha) y_{k-1}(t) ;
$$

here, the stochastic parameters $\alpha$ consist of independent and identically distributed Bernoulli random variables taking the values of 0 or 1 with

$$
\operatorname{prob}\{\alpha=1\}=\mathscr{E}\{\alpha\}=\bar{\alpha}, \quad 0 \leq \bar{\alpha} \leq 1,
$$

where $\bar{\alpha}$ are known constants and $\mathscr{E}\{\cdot\}$ stands for the mathematical expectation of $\{\cdot\}$. Obviously, $\alpha$ is independent of $u_{k}(t), x_{k}(t)$, and $y_{k}(t)$.

In this paper, we consider the following ILC algorithm:

$$
u_{k+1}(t)=(1-\gamma) u_{k}(t)+\gamma u_{0}(t)+\Gamma e_{k}(t+1),
$$

where $e_{k}(t)=y_{d}(t)-\tilde{y}_{k}(t)$ is the measurement error and $\Gamma$ is the learning gain matrix which is to be chosen. $0<\gamma<1$ is a forgetting factor.

Remark 4. As stated in [20], the forgetting factor is introduced to prevent the input from wandering too much initially. In addition, it may be allowed to vary with the iteration to further improve performance. We leave it fixed for simplicity of presentation.

The $\lambda$-norm will be used in this paper. It is defined by

$$
\|f(\cdot)\|_{\lambda}=\sup _{0 \leq t \leq T} \lambda^{t}\|f(t)\|, \quad 0<\lambda<1
$$

for a vector function $f:[0, T] \rightarrow R^{n}$.

\section{Convergence Analysis}

Before giving the main results, we first give the following lemma [18].

Lemma 5. Suppose a real positive series $\{a\}_{1}^{\infty}$ satisfies

$$
a_{n} \leq \beta_{1} a_{n-1}+\beta_{2} a_{n-2}+\cdots+\beta_{N} a_{n-N}+\varepsilon,
$$

where $\beta_{i}(i=1,2, \ldots, N), \varepsilon \geq 0$, and $\beta=\sum_{i=1}^{N} \beta_{i}<1$. Then the following holds:

$$
\lim _{n \rightarrow \infty} a_{n} \leq \frac{\varepsilon}{1-\rho} .
$$

Proof. The proof can be found in [21]. 
Then, the following theorem can be obtained which shows that the iterative learning control scheme given in (5) can guarantee the asymptotic convergence of the output error.

Theorem 6. For the linear system (1), under Assumptions 1-3, when the ILC algorithm (5) is used, if

$$
\begin{array}{r}
\|(1-\gamma) I-\bar{\alpha} \Gamma C B\|+(1-\bar{\alpha})\|\Gamma C B\| \leq \rho_{1}+\rho_{2}<1, \\
0 \leq t \leq T,
\end{array}
$$

then as $k \rightarrow \infty$, the expectation of input and output errors is bounded. These bounds depend on the bound on the $b_{w}, b_{v}, b_{x_{0}}$, and $\gamma$. As $b_{w}, b_{v}, b_{x_{0}}$, and $\gamma$ tend to zero, these bounds also tend to zero.

Proof. Denote $\Delta x_{k}(t)=x_{d}(t)-x_{k}(t), \Delta u_{k}(t)=u_{d}(t)-u_{k}(t)$ notice that

$$
\begin{gathered}
x_{d}(t+1)=A x_{d}(t)+B u_{d}(t), \\
x_{k}(t+1)=A x_{k}(t)+B u_{k}(t)+w_{k}(t) ;
\end{gathered}
$$

then,

$$
\Delta x_{k}(t+1)=A \Delta x_{k}(t)+B \Delta u_{k}(t)-w_{k}(t) .
$$

Similarily, we also can obtain

$$
\Delta y_{k}(t)=C \Delta x_{k}(t)-v_{k}(t),
$$

where $\Delta y_{k}(t)=y_{d}(t)-y_{k}(t)$.

Considering the control error at $k+1$ iteration and using (11) and (12), we have

$$
\begin{aligned}
\Delta u_{k+1}(t)= & u_{d}(t)-u_{k+1}(t) \\
= & u_{d}(t)-(1-\gamma) u_{k}(t)+\gamma u_{0}(t) \\
& -\Gamma\left(y_{d}(t+1)-\alpha y_{k}(t+1)-(1-\alpha) y_{k-1}(t+1)\right) \\
= & (1-\gamma) \Delta u_{k}(t)+\gamma \Delta u_{0}(t) \\
& -\Gamma\left(\alpha \Delta y_{k}(t+1)+(1-\alpha) \Delta y_{k-1}(t+1)\right) \\
= & (1-\gamma) \Delta u_{k}(t)+\gamma \Delta u_{0}(t)-\Gamma \alpha C \Delta x_{k}(t+1) \\
& -(1-\alpha) \Gamma C \Delta x_{k-1}(t+1) \\
& +\alpha \Gamma v_{k}(t+1)+(1-\alpha) \Gamma v_{k-1}(t+1) \\
= & ((1-\gamma) I-\Gamma \alpha C B) \Delta u_{k}(t) \\
& -(1-\alpha) \Gamma C B \Delta u_{k-1}(t)+\gamma \Delta u_{0}(t) \\
& -\Gamma \alpha C A \Delta x_{k}(t)-(1-\alpha) \Gamma C A \Delta x_{k-1}(t) \\
& +\alpha \Gamma C w_{k}(t)+(1-\alpha) \Gamma C w_{k-1}(t) \\
& +\alpha \Gamma v_{k}(t+1)+(1-\alpha) \Gamma v_{k-1}(t+1) .
\end{aligned}
$$

Taking the norm of both sides of (13) and making use of the general properties of norms, we can obtain that

$$
\begin{aligned}
\left\|\Delta u_{k+1}(t)\right\| \leq & \|(1-\gamma) I-\Gamma \alpha C B\|\left\|\Delta u_{k}(t)\right\| \\
& +\|(1-\alpha) \Gamma C B\|\left\|\Delta u_{k-1}(t)\right\|+\gamma\left\|\Delta u_{0}(t)\right\| \\
& +\|\alpha \Gamma C A\|\left\|\Delta x_{k}(t)\right\| \\
& +\|(1-\alpha) \Gamma C A\|\left\|\Delta x_{k-1}(t)\right\|+b_{w}\|\alpha \Gamma C\| \\
& +b_{w}\|(1-\alpha) \Gamma C\|+b_{v}\|\alpha \Gamma\| \\
& +b_{v}\|(1-\alpha) \Gamma\| .
\end{aligned}
$$

Taking expectation in both sides of (14), consider that $\alpha$ is independent of $u_{k}(t), x_{k}(t)$; we have

$$
\begin{aligned}
\mathscr{E}\{\| & \left.\Delta u_{k+1}(t) \|\right\} \\
\leq & \|(1-\gamma) I-\bar{\alpha} \Gamma C B\| \mathscr{E}\left\|\Delta u_{k}(t)\right\| \\
& +\|(1-\bar{\alpha}) \Gamma C B\| \mathscr{E}\left\|\Delta u_{k-1}(t)\right\| \\
& +\gamma\left\|\Delta u_{0}(t)\right\|+\bar{\alpha}\|\Gamma C A\| \mathscr{E}\left\|\Delta x_{k}(t)\right\| \\
& +\|(1-\bar{\alpha}) \Gamma C A\| \mathscr{E}\left\|\Delta x_{k-1}(t)\right\| \\
& +\bar{\alpha} b_{w}\|\Gamma C\|+b_{w}\|(1-\bar{\alpha}) \Gamma C\| \\
& +\bar{\alpha} b_{v}\|\Gamma\|+b_{v}\|(1-\bar{\alpha}) \Gamma\| \\
\leq & \rho_{1} \mathscr{E}\left\{\left\|\Delta u_{k}(t)\right\|\right\}+\rho_{2} \mathscr{E}\left\{\left\|\Delta u_{k-1}(t)\right\|\right\}+b_{1} \mathscr{E}\left\{\left\|\Delta x_{k}(t)\right\|\right\} \\
& +b_{2} \mathscr{E}\left\{\left\|\Delta x_{k-1}(t)\right\|\right\}+\gamma b_{u_{0}}+b_{w} d_{1}+b_{v} d_{2},
\end{aligned}
$$

where

$$
\begin{gathered}
\rho_{1}=\|(1-\gamma) I-\bar{\alpha} \Gamma C B\|, \quad \rho_{2}=(1-\bar{\alpha})\|\Gamma C B\|, \\
b_{1}=\bar{\alpha} \sup \{\|\Gamma C A\|\}, \quad b_{2}=\sup \{\|(1-\bar{\alpha}) \Gamma C A\|\}, \\
b_{u_{0}}=\sup \left\{\left\|\Delta u_{0}(t)\right\|\right\}, \\
d_{1}=\sup \{\bar{\alpha}\|\Gamma C\|+\|(1-\bar{\alpha}) \Gamma C\|\}, \\
d_{2}=\sup \{\bar{\alpha}\|\Gamma\|+\|(1-\bar{\alpha}) \Gamma\|\} .
\end{gathered}
$$

For any positive integer $t$, it can be calculated that

$$
\begin{array}{r}
x_{k}(t)=\Phi(t, 0) x_{k}(0)+\sum_{s=0}^{t-1} \Phi(t, s+1) B u_{k}(s), \\
(1 \leq t \leq T+1),
\end{array}
$$

where $\Phi(t, s)$ is defined as $\Phi(t, s)=A^{t-s}, \Phi(s, s)=I,(t>2)$.

From (17), we can obtain

$$
\Delta x_{k}(t)=\Phi(t, 0) \Delta x_{k}(0)+\sum_{s=0}^{t-1}\left(\Phi(t, s+1) B \Delta u_{k}(s)\right),
$$

where $\Delta x_{k}(0)=x_{d}(0)-x_{k}(0)$. 
From (15) and (18), the following can be easily obtained:

$$
\begin{aligned}
\mathscr{E}\left\{\left\|\Delta u_{k+1}(t)\right\|\right\} & \\
\leq & \rho_{1} \mathscr{E}\left\{\left\|\Delta u_{k}(t)\right\|\right\}+\rho_{2} \mathscr{E}\left\{\left\|\Delta u_{k-1}(t)\right\|\right\} \\
& +b_{1} \mathscr{E}\left\{\|\Phi(t, 0)\|\left\|\Delta x_{k}(0)\right\|\right. \\
& \left.+\left\|\sum_{s=0}^{t-1}\left(\Phi(t, s+1) B \Delta u_{k}(s)\right)\right\|\right\} \\
& +b_{2} \mathscr{E}\left\{\|\Phi(t, 0)\|\left\|\Delta x_{k-1}(0)\right\|\right. \\
& +\gamma b_{u_{0}}+b_{w} d_{1}+b_{v} d_{2} \\
\leq & \rho_{1} \mathscr{E}\left\{\left\|\Delta u_{k}(t)\right\|\right\}+\rho_{2} \mathscr{E}\left\{\left\|\Delta u_{k}(t)\right\|\right\} \\
& +\left(b_{1}+b_{2}\right) b_{\phi_{0}} b_{x_{0}}+\gamma b_{u_{0}}+b_{w} d_{1}+b_{v} d_{2}, \\
& +b_{1} b_{\phi} \sum_{s=0}^{t-1} \mathscr{E}\left\{\left\|\Delta u_{k}(s)\right\|\right\} \\
& +b_{2} b_{\phi} \sum_{s=0}^{t-1} \mathscr{E}\left\{\left\|\Delta u_{k-1}(s)\right\|\right\}
\end{aligned}
$$

where $b_{\phi_{0}}=\sup _{0 \leq t \leq T}\|\Phi(t, 0)\|, b_{\phi}=\sup _{0 \leq t \leq T, 0 \leq s \leq t-1} \| \Phi(t, s+$ 1) $B \|$. obtain

Multiplying both sides of (19) by $\lambda^{t}(0<\lambda<1)$, we can

$$
\begin{aligned}
& \mathscr{E}\left\{\lambda^{t}\left\|\Delta u_{k+1}(t)\right\|\right\} \\
& \leq \rho_{1} \mathscr{E}\left\{\lambda^{t}\left\|\Delta u_{k}(t)\right\|\right\} \\
&+b_{1} b_{\phi} \sum_{s=0}^{t-1} \lambda^{t-s} \mathscr{E}\left\{\lambda^{s}\left\|\Delta u_{k}(s)\right\|\right\}+\rho_{2} \mathscr{E}\left\{\lambda^{t}\left\|\Delta u_{k-1}(t)\right\|\right\} \\
&+b_{2} b_{\phi} \sum_{s=0}^{t-1} \lambda^{t-s} \lambda^{s} \mathscr{E}\left\{\left\|\Delta u_{k-1}(s)\right\|\right\}+\varsigma \\
& \leq \rho_{1} \mathscr{E}\left\{\lambda^{t}\left\|\Delta u_{k}(t)\right\|\right\} \\
&+b_{1} b_{\phi} \sum_{s=0}^{t-1} \lambda^{t-s} \cdot \mathscr{E}\left\{\sup _{0 \leq \tau \leq T}\left\{\lambda^{\tau}\left\|\Delta u_{k}(\tau)\right\|\right\}\right\} \\
&+\rho_{2} \mathscr{E}\left\{\lambda^{t}\left\|\Delta u_{k-1}(t)\right\|\right\} \\
&+b_{2} b_{\phi} \sum_{s=0}^{t-1} \lambda^{t-s} \cdot \mathscr{E}\left\{\sup _{0 \leq \tau \leq T}\left\{\lambda^{\tau}\left\|\Delta u_{k-1}(\tau)\right\|\right\}\right\}+\varsigma
\end{aligned}
$$

where $\varsigma=\left(b_{1}+b_{2}\right) b_{\phi_{0}} b_{x_{0}}+\gamma b_{u_{0}}+b_{w} d_{1}+b_{v} d_{2}$; then

$$
\begin{aligned}
\mathscr{E}\left\{\left\|\Delta u_{k+1}(t)\right\|_{\lambda}\right\} \leq & \bar{\rho}_{1} \mathscr{E}\left\{\left\|\Delta u_{k}(t)\right\|_{\lambda}\right\} \\
& +\bar{\rho}_{2} \mathscr{E}\left\{\left\|\Delta u_{k-1}(t)\right\|_{\lambda}\right\}+\varsigma
\end{aligned}
$$

where $\bar{\rho}_{1}=\rho_{1}+\bar{\alpha} b_{1} b_{\phi}\left(\lambda\left(1-\lambda^{T}\right) /(1-\lambda)\right), \bar{\rho}_{2}=\rho_{2}+(1-$ $\bar{\alpha}) b_{1} b_{\phi}\left(\lambda\left(1-\lambda^{T}\right) /(1-\lambda)\right)$.

Obviously, since $\rho_{1}+\rho_{2}<1$, we can find a sufficiently small $\lambda$ such that

$$
\begin{aligned}
\bar{\rho}_{1}+\bar{\rho}_{2}= & \rho_{1}+\rho_{2}+b_{1} b_{\phi} \frac{\lambda\left(1-\lambda^{T}\right)}{1-\lambda} \\
& +b_{2} b_{\phi} \frac{\lambda\left(1-\lambda^{T}\right)}{1-\lambda}<1
\end{aligned}
$$

then, using Lemma 5 and the inequality (21), we have

$$
\lim _{k \rightarrow \infty} \mathscr{E}\left\{\left\|\Delta u_{k}(t)\right\|_{\lambda}\right\} \leq \frac{\varsigma}{1-\left(\bar{\rho}_{1}+\bar{\rho}_{2}\right)} .
$$

From (12) and (18), we can obtain

$$
\begin{aligned}
& \mathscr{E}\left\{\lambda^{t}\left\|\Delta y_{k}(t)\right\|\right\} \\
& \leq\|C\| \mathscr{E}\left\{\lambda^{t}\left\|\Delta x_{k}(t)\right\|\right\}+b_{v} \\
& \leq\|C\| \mathscr{E}\left\{\|\Phi(t, 0)\|\left\|\Delta x_{k}(0)\right\|\right. \\
& \left.\quad+\lambda^{t}\left\|\sum_{s=0}^{t-1}\left(\Phi(t, s+1) B \Delta u_{k}(s)\right)\right\|\right\}+b_{v} \\
& \leq b_{c} b_{\phi} \sum_{s=0}^{t-1} \lambda^{t-s} \mathscr{E}\left\{\lambda^{s}\left\|\Delta u_{k}(s)\right\|\right\}+b_{c} b_{\phi_{0}} b_{x_{0}}+b_{v} \\
& \leq b_{c} b_{\phi} \frac{\lambda\left(1-\lambda^{T}\right)}{1-\lambda}\left\|\Delta u_{k}(t)\right\|_{\lambda}+b_{c} b_{\phi_{0}} b_{x_{0}}+b_{v},
\end{aligned}
$$


where $b_{c}=\sup \|C\|$. That is,

$$
\begin{aligned}
\mathscr{E}\left\{\left\|\Delta y_{k}(t)\right\|_{\lambda}\right\} \leq & b_{c} b_{\phi} \frac{\lambda\left(1-\lambda^{T}\right)}{1-\lambda}\left\|\Delta u_{k}(t)\right\|_{\lambda} \\
& +b_{c} b_{\phi_{0}} b_{x_{0}}+b_{v} \\
\leq & b_{c} b_{\phi} \frac{\lambda\left(1-\lambda^{T}\right)}{1-\lambda} \frac{\varsigma}{1-\left(\bar{\rho}_{1}+\bar{\rho}_{2}\right)} \\
& +b_{c} b_{\phi_{0}} b_{x_{0}}+b_{v},
\end{aligned}
$$

and (24) and (25) mean that the expectation of input and output errors is bounded as $k \rightarrow \infty$; these bounds depend on the bound on the $b_{w}, b_{v}, b_{x_{0}}$, and $\gamma$. As $b_{w}, b_{v}, b_{x_{0}}$, and $\gamma$ tend to zero, these bounds also tend to zero.

This completes the proof.

Remark 7. In this paper, we consider the intermittent measurement for linear ILC systems. From the proof of Theorem 6, it is easy to see that the method proposed can be extended to solving problems in other frameworks such as sensor delay, input signal dropouts and and the system can also be extended to linear time varying systems. Besides, if the ILC algorithm is chosen as $u_{k+1}(t)=u_{k}(t)+\Gamma\left(e_{k}(t+1)+e_{k}(t)\right)$, the similar result can also be obtained.

Remark 8. In many applications, we desire to stop the process in a finite time, and we desire the error to be as small as possible. In this case, the forgetting factor $\gamma$ is helpful, and varying operator as the iterations progress may improve performance. The bias term is initially useful to keep the input from wandering excessively. Once the input has converged fairly well, we may want to begin decreasing the learning gain to cause the input to average out random disturbances, thus improving the accuracy of the final input. It is easily seen that these modifications do not change the results of Theorem 6 .

\section{Numerical Example}

In this section, an example is given to verify our conclusions. Consider the following linear system:

$$
\begin{gathered}
x_{k}(t+1)=\left[\begin{array}{cc}
-0.8 & -0.22 \\
1 & 0
\end{array}\right] x_{k}(t)+\left[\begin{array}{c}
0.5 \\
1
\end{array}\right] u_{k}(t)+w_{k}(t), \\
y_{k}(t)=\left[\begin{array}{ll}
1 & 0.5
\end{array}\right] x_{k}(t)+v_{k}(t) .
\end{gathered}
$$

The desired trajectory is

$$
y_{d}(t)=2 \cos \left(\frac{\pi t}{300}\right), \quad t \in[0,1000]
$$

We assume $\bar{\alpha}=0.75$, and that means $25 \%$ packet dropouts. The learning gain in (5) is given as $\Gamma=0.2$. To check

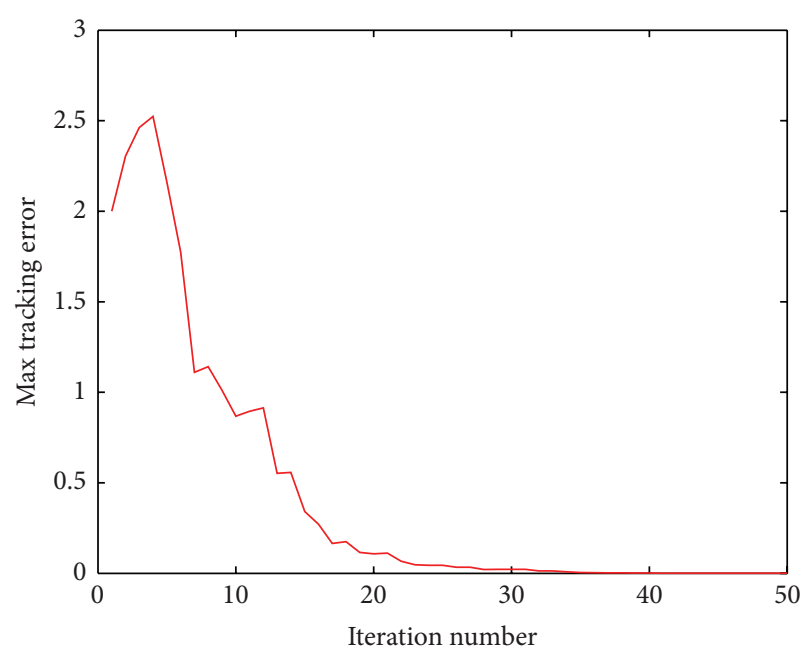

FIGURE 1: The max tracking error versus iteration number for case 1.

the tracking performance of the ILC law, we consider the following three cases.

$$
\begin{aligned}
& \text { Case 1: } x_{k}(0)=x_{d}(0)=\left[\begin{array}{ll}
1 & 0
\end{array}\right]^{T}, w_{k}(t)=0, v_{k}(t)=0, \\
& \gamma=0 . \\
& \text { Case 2: }\left\|x_{k}(0)-x_{d}(0)\right\| \in[0,0.05], w_{k}(t) \in[0,0.05], \\
& v_{k}(t) \in[0,0.05], \gamma=0 . \\
& \text { Case 3: }\left\|x_{k}(0)-x_{d}(0)\right\| \in[0,0.05], w_{k}(t) \in[0,0.05], \\
& v_{k}(t) \in[0,0.05], \gamma=0.2 .
\end{aligned}
$$

Case 1 means that the intermittent ILC system does not contain any initial state error and disturbances. In this case, checking the condition in Theorem 6 , we can obtain $\|(1-\gamma) I-$ $\bar{\alpha} \Gamma C B\|+(1-\bar{\alpha})\| \Gamma C B \|=0.88<1$; the intermittent ILC system is stable. Due to $x_{k}(0)=x_{d}(0), w_{k}(t)=0, v_{k}(t)=0, \gamma=0$, the tracking error satisfies $\lim _{k \rightarrow \infty} \mathscr{E}\left\{\Delta y_{k}(t)\right\}=0$. In order to obtain an estimate of $\mathscr{E}\left\{\Delta y_{k}(t)\right\}$, the simulation is repeated 50 times, and the expectation operator is implemented as a mean over the simulations. The simulation results are shown in Figures 1 and 2. As shown in Figure 1, the maximum tracking error goes to zero as iteration increases when no initial state error and disturbances exist. Figure 2 gives the system output profiles at different iterations. Obviously, the system tracking error converges from iteration to iteration. However, the convergence is not monotonic but is asymptotic. Case 2 means that the intermittent ILC system contains initial state error and disturbances. Simulation results for this case are shown in Figures 3 and 4. It is observed that the tracking error cannot converge to zero, and the bounded error is caused by initial state error and bounded disturbances. To improve the performance of the ILC system, the robust ILC with a forgetting factor is introduced in case 3 . In this case, the condition of Theorem 6 is $\|(1-\gamma) I-\bar{\alpha} \Gamma C B\|+(1-\bar{\alpha})\|\Gamma C B\|=$ $0.68<1$, and then the system is stable. Figures 5 and 6 give the tracking errors and system output profiles at different iterations, respectively. Comparing Figure 3 with Figure 5, we observe that the better tracking performance can be obtained when the robust ILC law is used. 


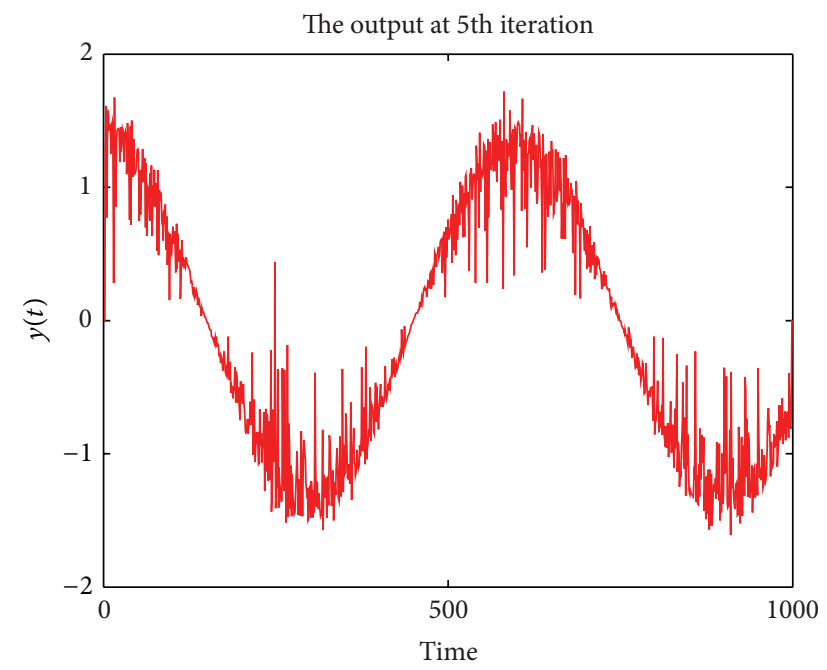

(a)

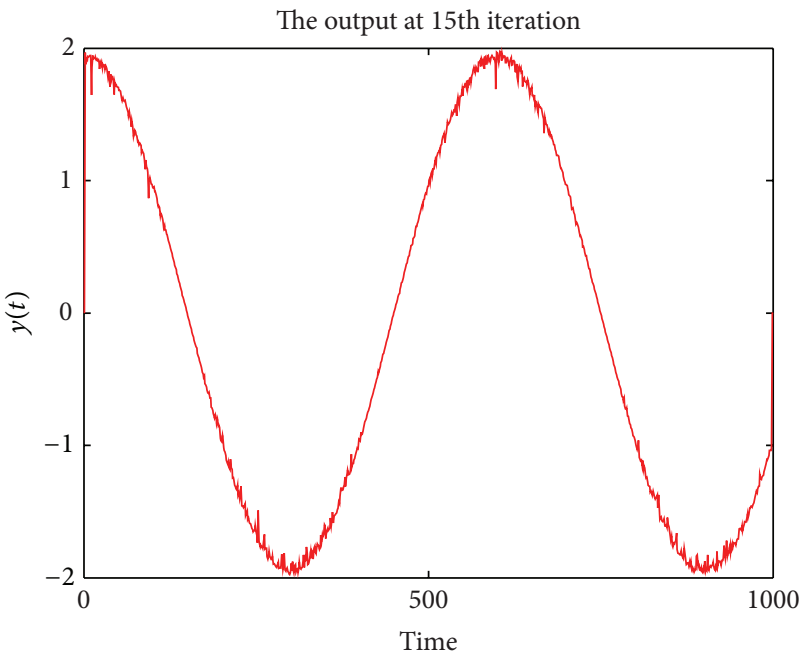

(c)

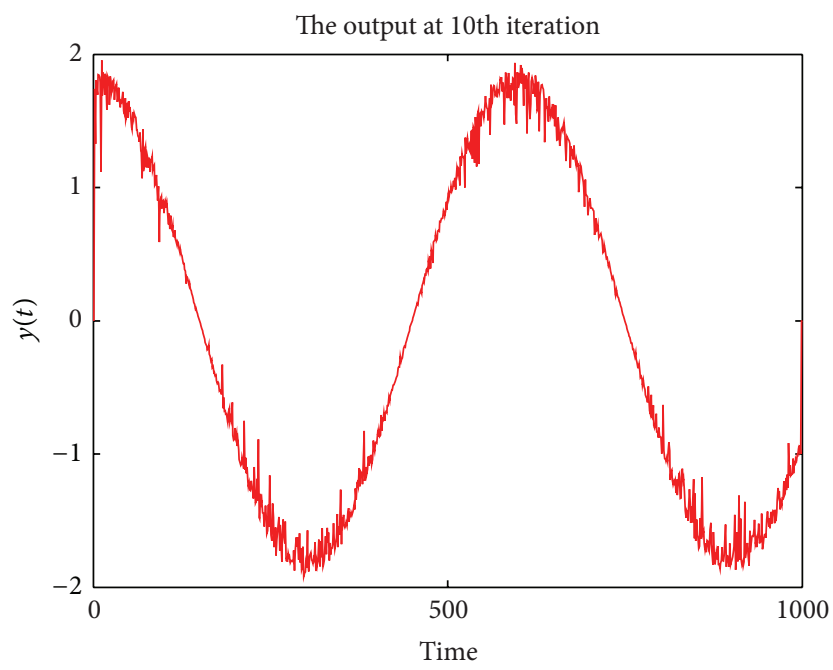

(b)

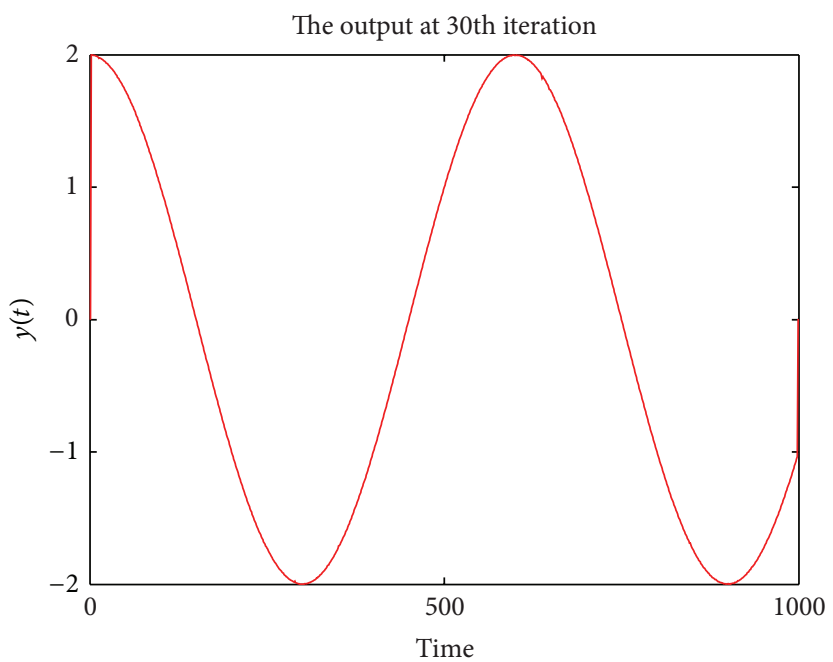

(d)

Figure 2: The system outputs of different iterations for case 1.

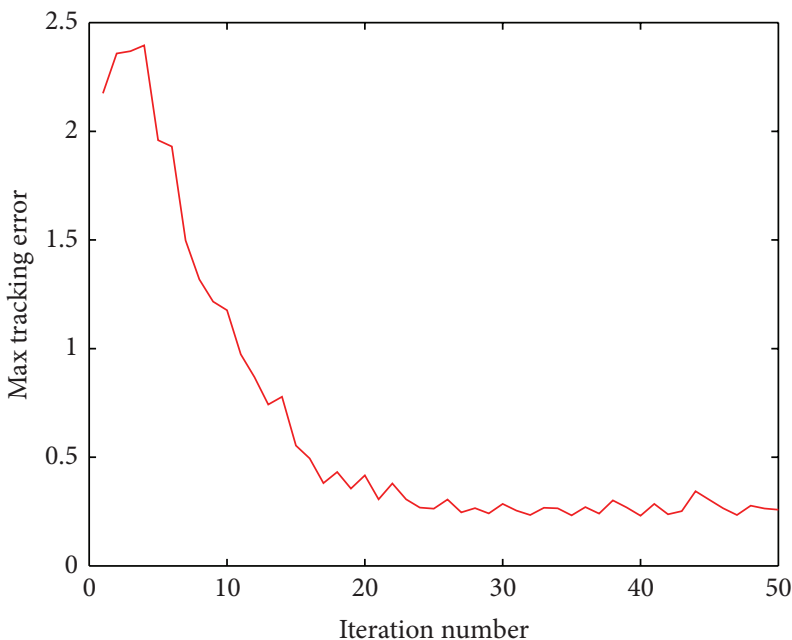

FIGURE 3: The max tracking error versus iteration number for case 2. 


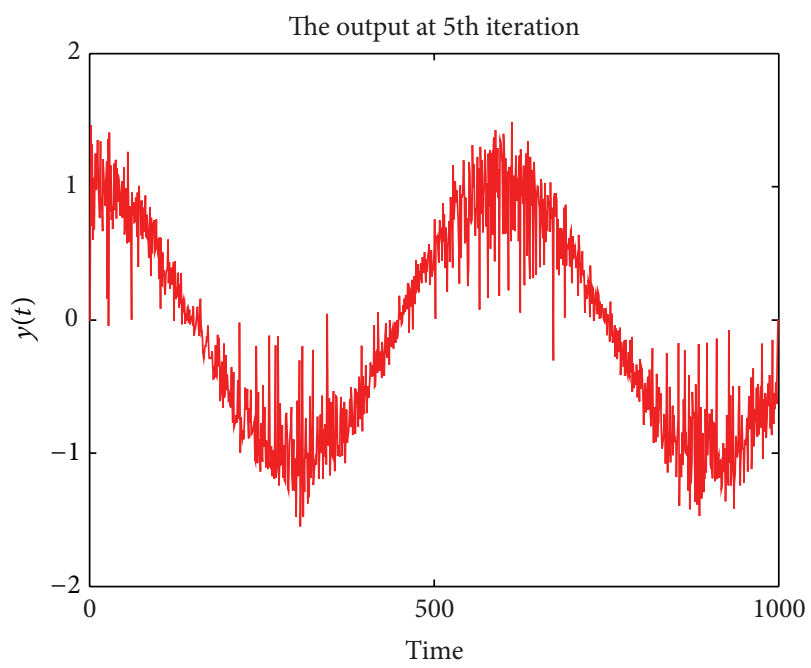

(a)

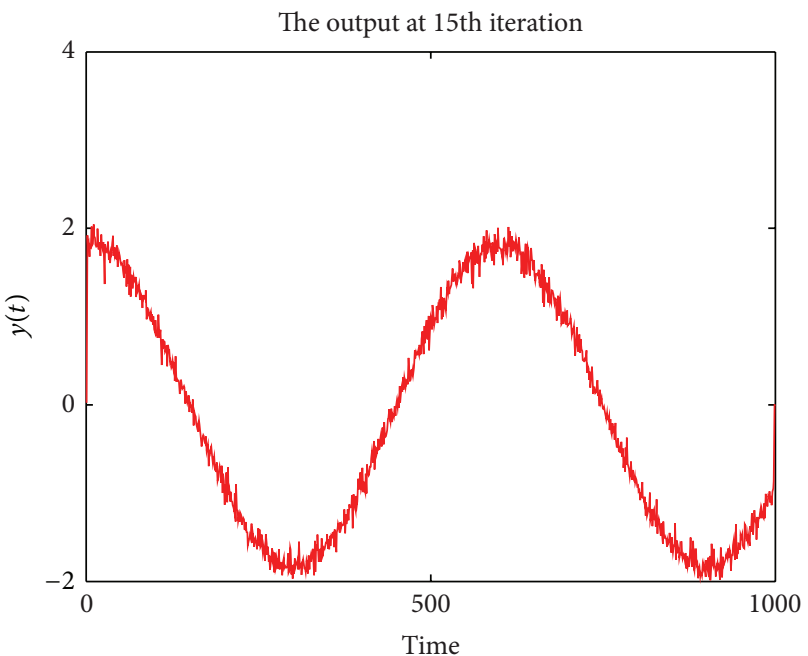

(c)

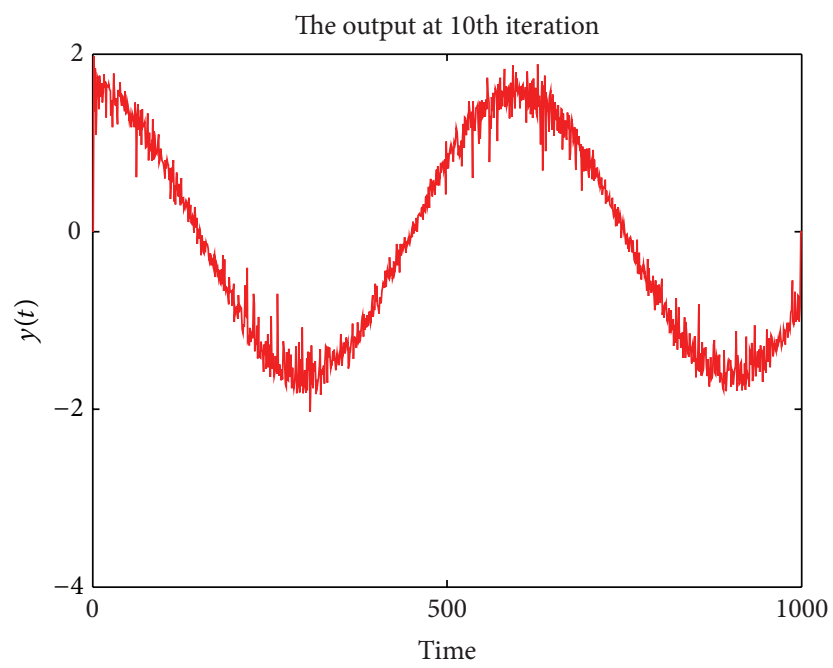

(b)

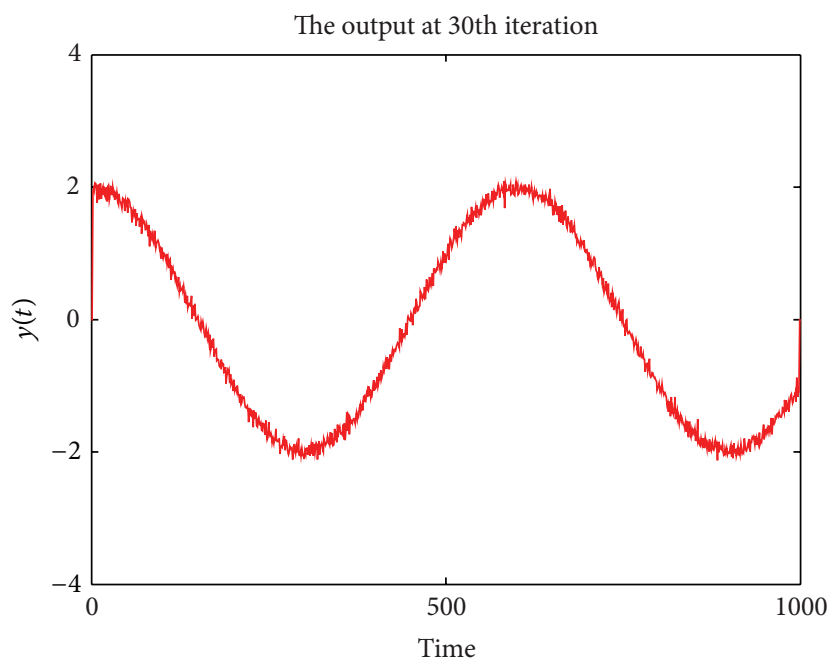

(d)

FIgURE 4: The system outputs of different iterations with for case 2.

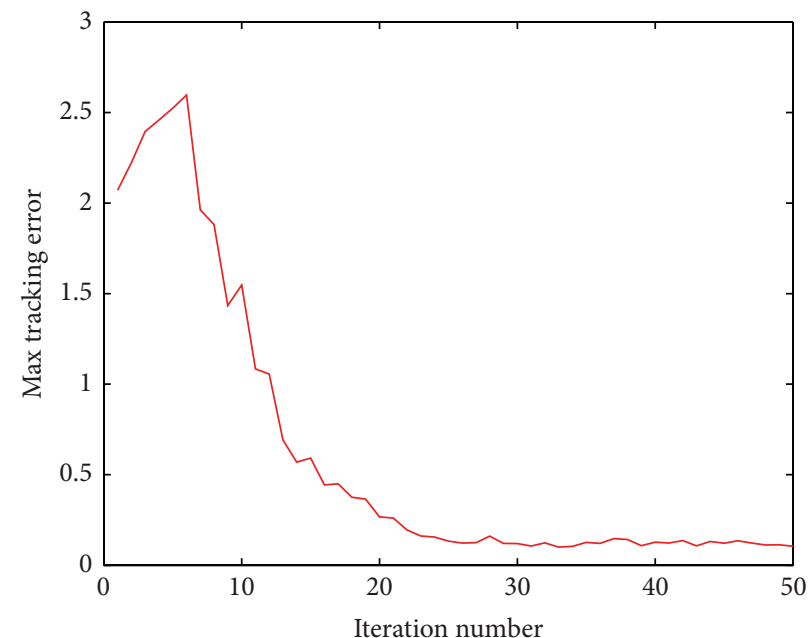

FIGURE 5: The max tracking error versus iteration number for case 3. 


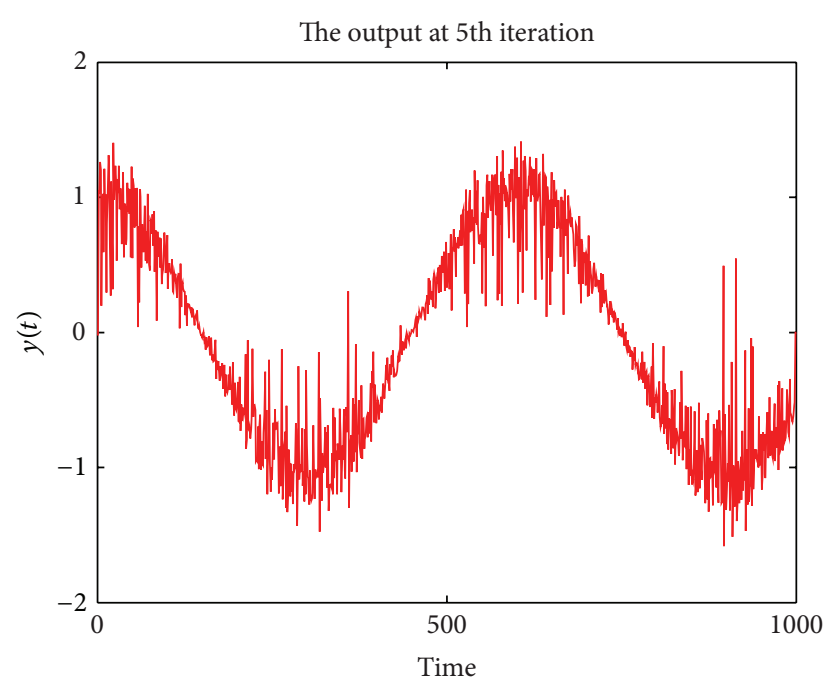

(a)

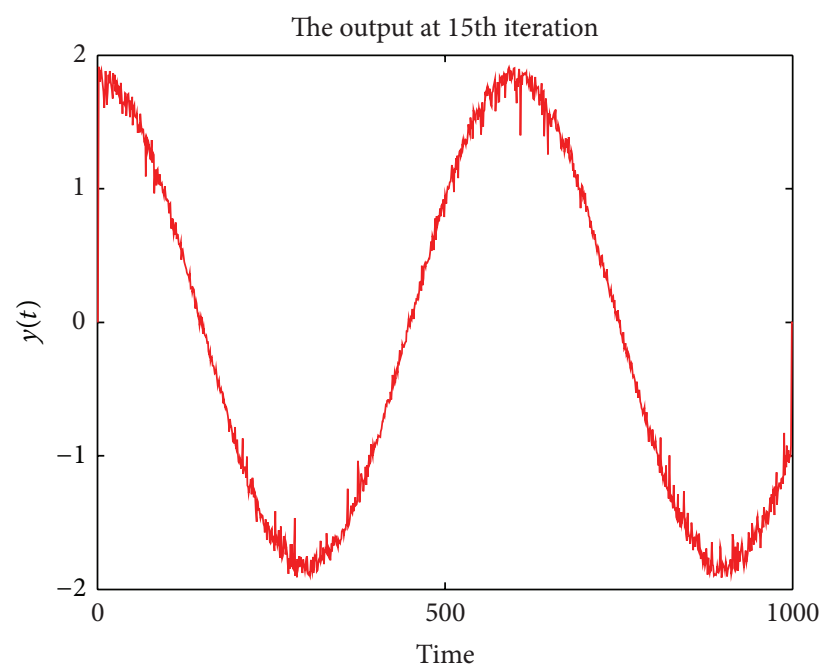

(c)

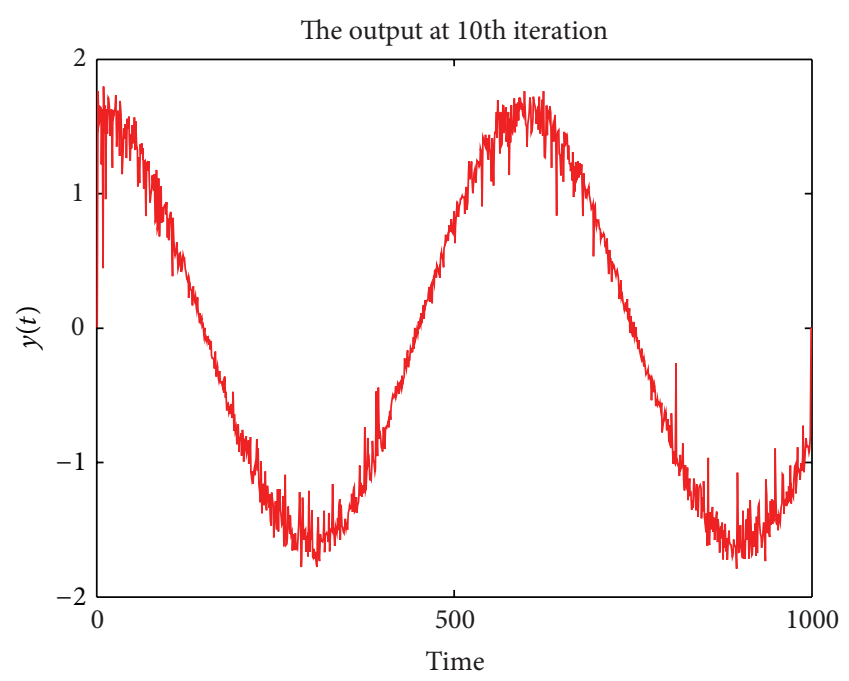

(b)

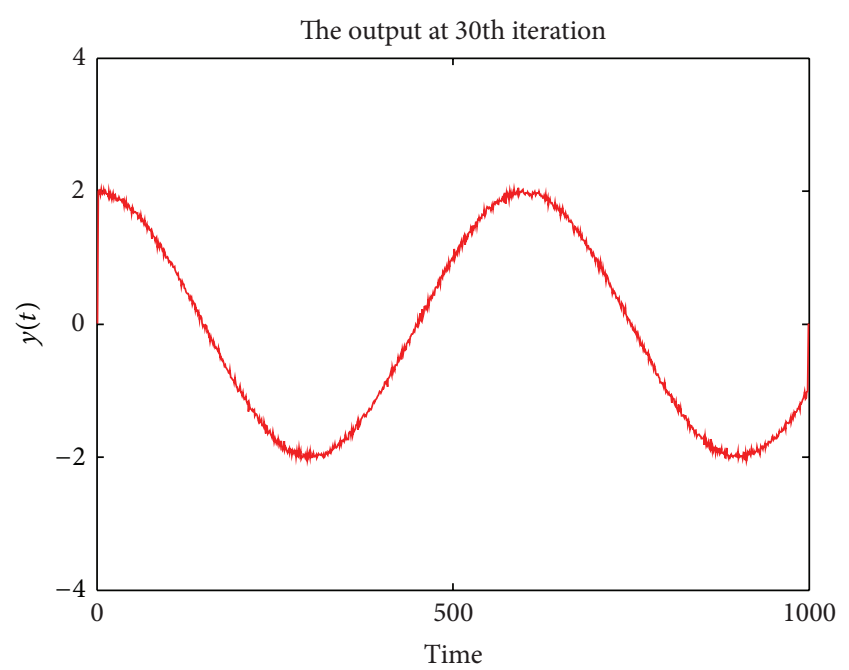

(d)

FIgURE 6: The system outputs of different iterations for case 3.

\section{Conclusions}

In this paper, the robust iterative learning control design for a class of intermittent systems with disturbances and uncertain initial conditions is considered. It is shown that the robust convergence of the intermittent ILC system can be guaranteed, and the asymptotic trajectory errors for the control input and output trajectories are bounded. These bounds depend on bounds on the initial condition errors and disturbances, and the bounds are zero in the absence of these disturbances. Simulation results show that the robust iterative learning control can attenuate these bounds effectively. In future work, we will discuss the proposed ILC algorithm for nonlinear systems and design robust learning gains to improve the transient behavior of the intermittent ILC systems.

\section{Acknowledgments}

This work was supported by the National Science Foundation of China (61203065), the Program of Natural Science of Henan Provincial Education Department (12A510013, 12B520020), and the Program of Open Laboratory Foundation of Control Engineering Key Discipline of Henan Provincial High Education (KG 2011-10).

\section{References}

[1] S. Arimoto, S. Kawamura, and F. Miyazaki, "Bettering operation of robots by learning," Journal of Robotic Systems, vol. 1, no. 2, pp. 123-140, 1984.

[2] Z. Bien and J. X. Xu, Iterative Learning Control: Analysis, Design, Integration and Applications, Kluwer Academic Publishers, Dordrecht, The Netherlands, 1998. 
[3] H.-S. Ahn, Y. Q. Chen, and K. L. Moore, "Iterative learning control: brief survey and categorization," IEEE Transactions on Systems, Man and Cybernetics Part C, vol. 37, no. 6, pp. 10991121, 2007.

[4] D. A. Bristow, M. Tharayil, and A. G. Alleyne, "A survey of iterative learning control: a learning-based method for highperformance tracking control," IEEE Control Systems Magazine, vol. 26, no. 3, pp. 96-114, 2006.

[5] R. Chi, Z. Hou, and J. Xu, "Adaptive ILC for a class of discretetime systems with iteration-varying trajectory and random initial condition," Automatica, vol. 44, no. 8, pp. 2207-2213, 2008.

[6] M. Sun and D. Wang, "Initial shift issues on discrete-time iterative learning control with system relative degree," IEEE Transactions on Automatic Control, vol. 48, no. 1, pp. 144-148, 2003.

[7] T. Wang, S. Tong, and Y. Li, "Robust adaptive fuzzy control for nonlinear system with dynamic uncertainties based on backstepping," International Journal of Innovative Computing, Information and Control, vol. 5, no. 9, pp. 2675-2688, 2009.

[8] S. Tong, W. Wang, and L. Qu, "Decentralized robust control for uncertain T-S fuzzy large-scale systems with time-delay," International Journal of Innovative Computing, Information and Control, vol. 3, no. 3, pp. 657-672, 2007.

[9] S. Tong, C. Liu, and Y. Li, "Fuzzy-adaptive decentralized output-feedback control for large-scale nonlinear systems with dynamical uncertainties," IEEE Transactions on Fuzzy Systems, vol. 18, no. 5, pp. 845-861, 2010.

[10] W. Zhang, M. S. Branicky, and S. M. Phillips, "Stability of networked control systems," IEEE Control Systems Magazine, vol. 21, no. 1, pp. 84-97, 2001.

[11] P. Seiler and R. Sengupta, "An $H_{\infty}$ approach to networked control," IEEE Transactions on Automatic Control, vol. 50, no. 3, pp. 356-364, 2005.

[12] J. Wu and T. Chen, "Design of networked control systems with packet dropouts," IEEE Transactions on Automatic Control, vol. 52, no. 7, pp. 1314-1319, 2007.

[13] Q. Ling and M. D. Lemmon, "Power spectral analysis of networked control systems with data dropouts," IEEE Transactions on Automatic Control, vol. 49, no. 6, pp. 955-960, 2004.

[14] H.-S. Ahn, Y. Chen, and K. L. Moored, "Intermittent iterative learning control," in Proceedings of the IEEE International Symposium on Intelligent Control (ISIC '06), pp. 832-837, October 2006.

[15] H.-S. Ahn, K. L. Moore, and Y. Chen, "Discrete-time intermittent iterative learning controller with independent data dropouts," in Proceedings of the 17th World Congress, International Federation of Automatic Control (IFAC '08), pp. 1244212447, July 2008.

[16] C. Liu, J. Xu, and J. Wu, "Iterative learning control for network systems with communication delay or data dropout," in Proceedings of the 48th IEEE Conference on Decision and Control, pp. 4858-4863, China, December 2009.

[17] X.-H. Bu and Z.-S. Hou, "Stability of iterative learning control with data dropouts via asynchronous dynamical system," International Journal of Automation and Computing, vol. 8, no. 1, pp. 29-36, 2011.

[18] X. Bu, Z. Hou, and F. Yu, "Stability of first and high order iterative learning control with data dropouts," International Journal of Control, Automation and Systems, vol. 9, no. 5, pp. 843-849, 2011.

[19] X. H. Bu, F. S. Yu, Z. S. Hou, and F. Z. Wang, "Iterative learning control for a class of nonlinear systems with random packet losses," Nonlinear Analysis: Real World Applications, vol. 14, no. 1, pp. 567-580, 2013.

[20] G. Heinzinger, D. Fenwick, B. Paden, and F. Miyazaki, "Stability of learning control with disturbances and uncertain initial conditions," IEEE Transactions on Automatic Control, vol. 37, no. 1, pp. 110-114, 1992.

[21] Y. Chen, Z. Gong, and C. Wen, "Analysis of a high-order iterative learning control algorithm for uncertain nonlinear systems with state delays," Automatica, vol. 34 , no. 3, pp. 345353, 1998 . 


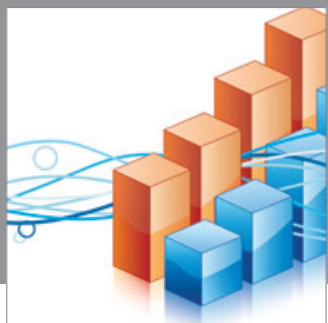

Advances in

Operations Research

mansans

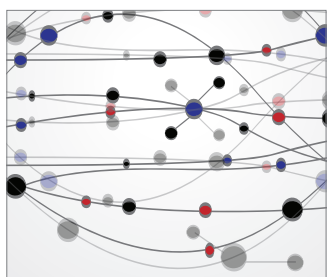

The Scientific World Journal
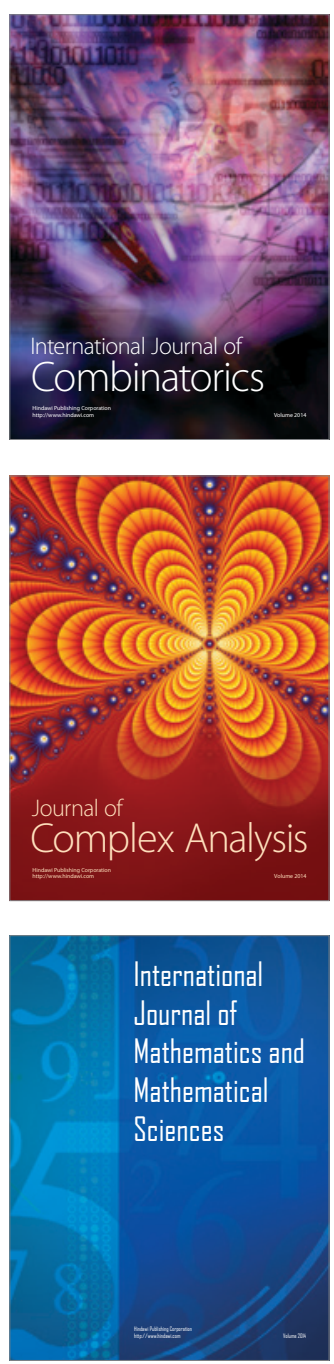
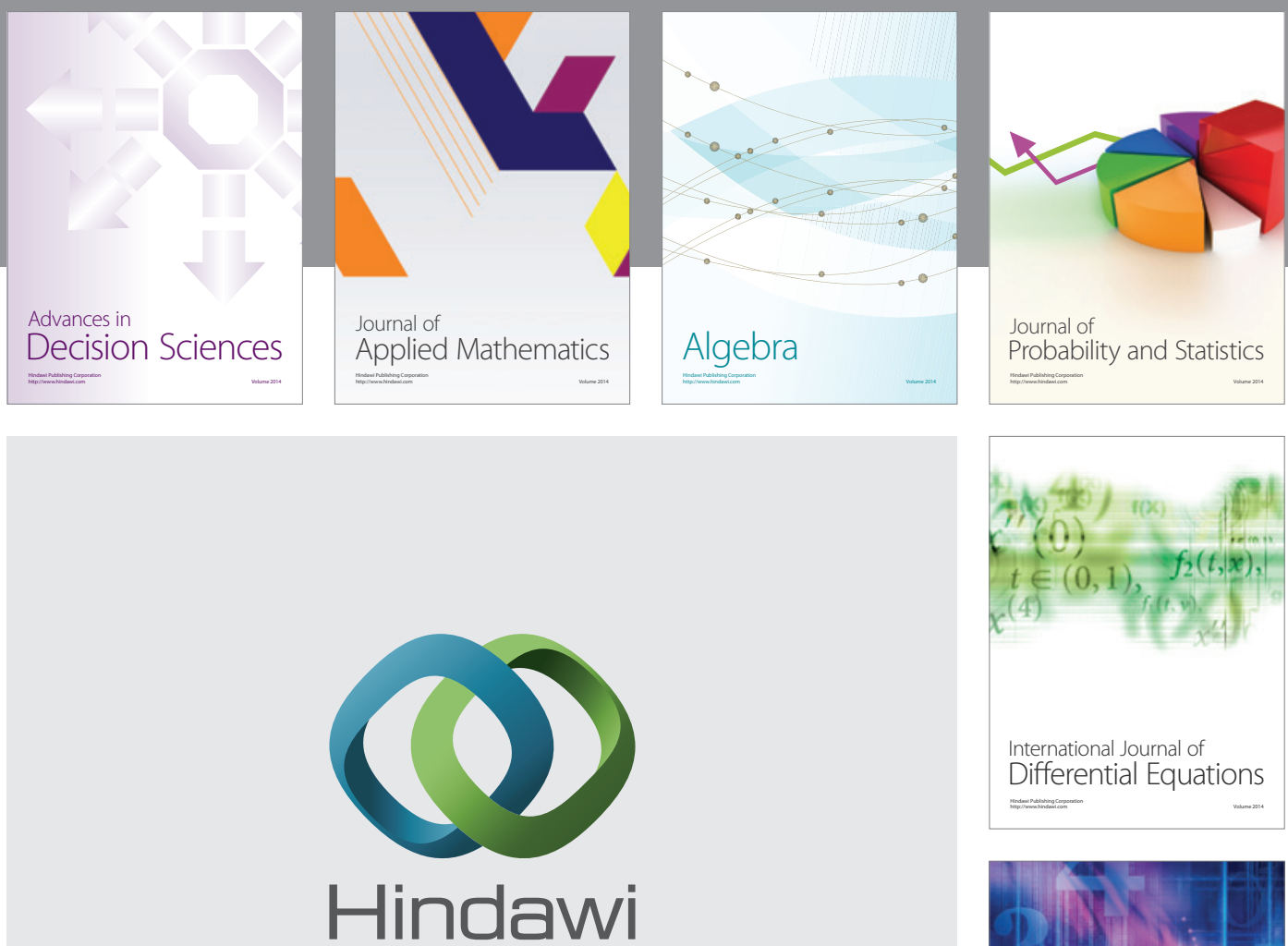

Submit your manuscripts at http://www.hindawi.com
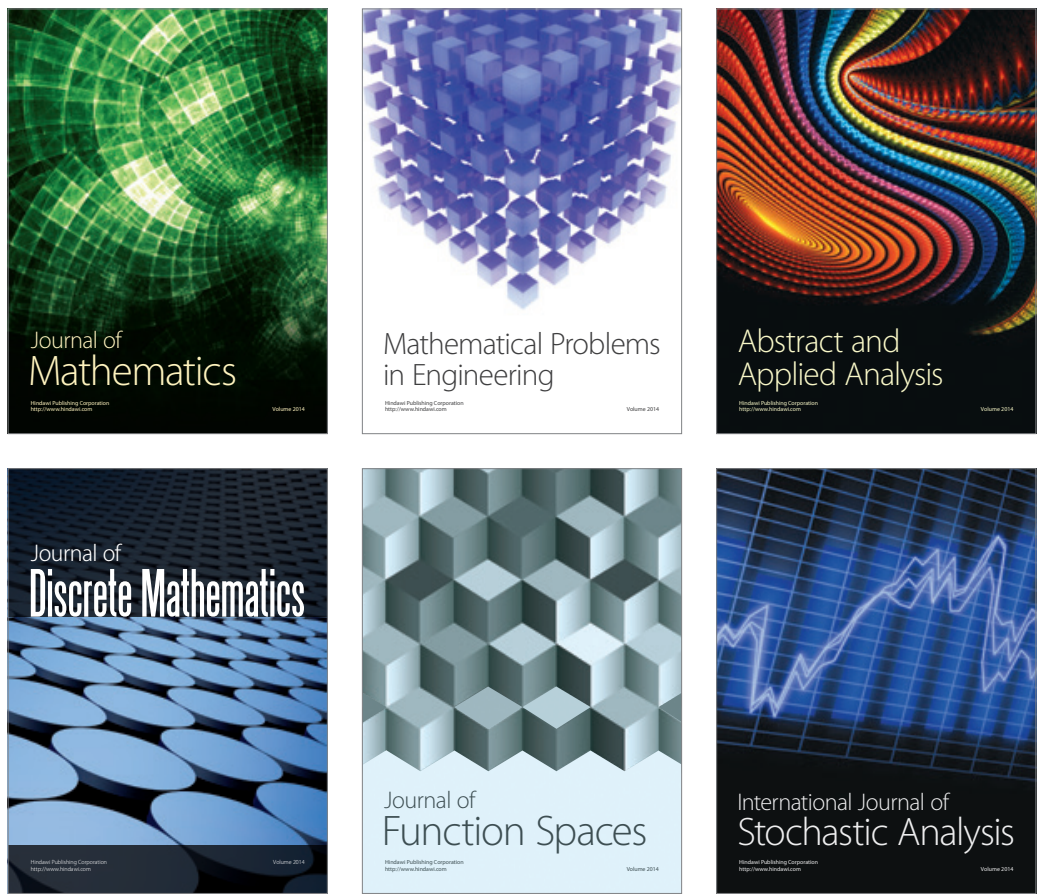

Journal of

Function Spaces

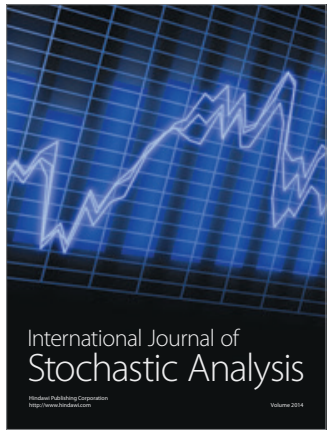

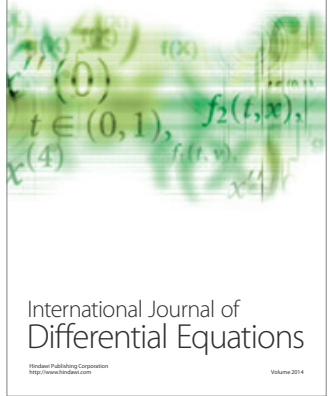
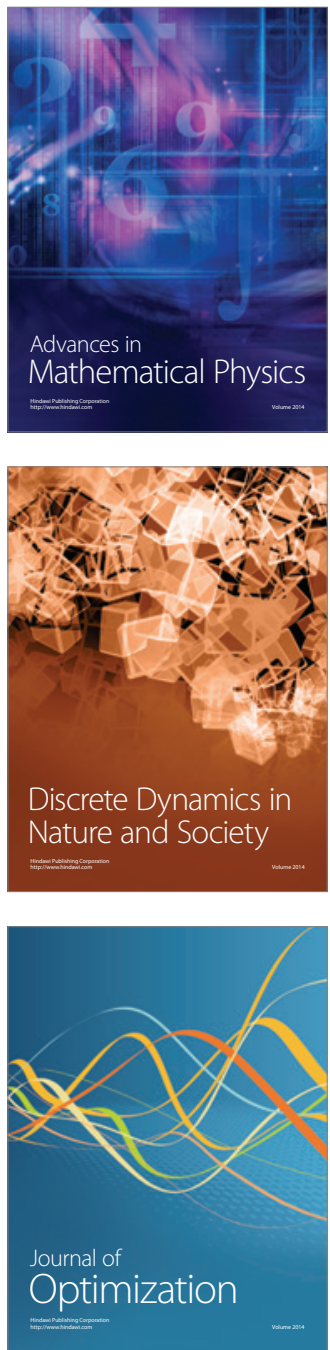